\title{
Diferencias de género y cambios tecnológicos en el campo laboral de la administración pública de Ecuador
}

\author{
Mónica C. Gallegos-Varela*, Ligia I. Beltrán-Urvina, Henry M. Vallejos-Orbe y Nelly E. Galiano-Andrade \\ Universidad Técnica del Norte, Facultad de Ciencias Administrativas y Económicas, Campus. Avenida 17 de Julio, \\ 5-21, General José María Córdova, Código postal 100105, Ibarra, Ecuador. (Correo-e: mgallegos@utn.edu.ec, \\ libeltran@utn.edu.ec, hmvallejos@utn.edu.ec,negaliano@utn.edu.ec)
}

* Autor a quien debe ser dirigida la correspondencia.

Recibido Abr. 28, 2021; Aceptado Jun. 29, 2021; Versión final Ago. 17, 2021, Publicado Dic. 2021

\begin{abstract}
Resumen
El principal objetivo de esta investigación es evaluar el nivel de competencias digitales de directivos de gobiernos autónomos descentralizados de Ecuador en torno al conocimiento y manejo de herramientas digitales básicas para su gestión en función de género y sus actitudes hacia las TIC (Tecnologías de la información y comunicación). Se emplea una metodología cuantitativa no experimental, descriptiva e inferencial. Se utiliza un cuestionario digital para la recolección de información a 85 directivos. Los resultados muestran diferencias no significativas en las competencias digitales de uso y conocimiento en función del género, así como una actitud positiva hacia las TIC tanto en hombres como en mujeres. Se evidencia que los hombres se valoran mejor que las mujeres en conocimientos, manejo y actitud. Se concluye que las diferencias de género no son significativas y se evidencia un progreso considerable por parte del género femenino en cuanto a las brechas entre hombres y mujeres.
\end{abstract}

\section{Gender differences and technological changes in Ecuador's public administration}

\begin{abstract}
The main objective of this research study is to assess digital competence among managers at the decentralized autonomous government in Ecuador in regards to knowledge and use of basic management digital tools according to gender and attitudes towards ICT (Information and communications technology). The methodology applied is quantitative, non-experimental, descriptive, and inferential. A digital questionnaire is used to survey 85 managers. The results show non-significant differences in digital skills and knowledge between genders. There is a positive attitude towards ICT in both men and women. Men are valued better than women in knowledge, management, and attitude. It is concluded that differences between genders are not significant and there is evidence of female progress in reducing gender gaps with males.
\end{abstract}

Keywords: digital skills; public administration; gender differences; ICT 


\section{INTRODUCCIÓN}

Las TIC exigen habilidades y competencias tanto de manera individual como a nivel de organizaciones, públicas o privadas, cada vez el campo laboral es más exigente en donde el género, mentalidad y -en fin- la cultura se exponen a cambios (Trenerry et al., 2021). En la administración pública las TIC son necesarias para promover su incorporación al servicio de los demás, pues necesariamente los estilos de gestión están cambiando, por mencionar, la toma de decisiones, la cooperación, el trabajo en equipo, la participación; los gerentes buscan cumplir los objetivos organizacionales con estrategias eficaces que ayuden a mejorar su desempeño y redunde en la satisfacción interna y externa; lo que significa -sin importar el género- un crecimiento en el nivel de capacidades y habilidades (Khalayleh et al., 2017).

Para Al-Omari et al., (2020) y (Al-Maani et al 2020) en cuanto a los procesos de planificación y gestión estratégica aseveran que el género y el liderazgo administrativo permiten obtener beneficios en el recurso humano, en su desempeño y satisfacción laboral, logrando aumentar y desarrollar las capacidades y habilidades de las personas. Es importante mencionar que el talento humano se concibe como la base de todos los procedimientos que contribuyen al éxito organizacional y la supervivencia en ámbitos competitivos, es uno de los factores que más influye a la hora de establecer diferencias sobre todo con relación a las TIC y que básicamente estas diferencias gravitan en la confianza, uso de las tecnologías digitales, actitudes ante las TIC, grado de conocimientos y habilidades que poseen (Cateriano et al., 2021).

Las instituciones públicas responden a estructuras organizacionales basadas a una estructura jerárquica y la separación de funciones. Toda organización conjetura un orden y estructura organizativa que da lugar a diferentes procesos que conducen al cumplimiento de objetivos, por lo tanto, la presencia de las competencias digitales debe contribuir, a obtener pirámides organizacionales que fortalezcan las capacidades directivas, de gestión y producción de forma transversal en todo el eje laboral del sector público. Para que una persona pueda cumplir eficientemente determinada tarea, necesita ciertas habilidades, capacidades y conocimientos. Esto se podría definir como competencia, misma que es adquirida mediante el aprendizaje y la formación (Abreu-Valdivia et al., 2020). Estas competencias digitales implican el uso crítico y seguro de las TIC para el trabajo, el tiempo libre y la comunicación, las cuales permiten integrar un trabajo colaborativo, que potencia habilidades (Levano-Francia, 2019). Investigaciones como la de Gilch y Sieweke (2021), han demostrado que las habilidades digitales a través del uso de las TIC están condicionadas por el género y que la brecha digital de género persiste más allá del acceso y usos básicos de las TIC, perpetuándose en otras dimensiones como la autonomía, experiencia, habilidad y tipos de uso (Rebollo-Catalán et al., 2017).

De acuerdo a estudios de (Sebastian y Ayuso, 2012) la incorporación de las mujeres en la gestión de las tecnologías de información ha sido un factor estratégico de suma importancia, desde una perspectiva competitiva y de bienestar social, lo que ha logrado su desarrollo y participación en empleos cualificados, negocios y ámbitos investigativos, por otro lado, investigadores como Pérez et al., (2021) aseguran que las mujeres poseen habilidades más marcadas en la creación de contenidos, demostrando mayor creatividad y predisposición es estos campos. Sin embargo, para Cabezas et al., (2017) y Vasilescu (2020) la influencia de la variable género en la adquisición de competencia digital si posee diferencias significativas y han verificado que los hombres se valoran con mayores habilidades digitales por encima de las mujeres en cuanto a conocimientos y actitud. Es así que, en esta investigación se han formulado las siguientes hipótesis: a) Los hombres poseen mayor conocimiento y manejo de las TIC que las mujeres y b) Los hombres poseen una mejor actitud hacia las TIC que las mujeres.

En el caso de las mujeres existe un mayor interés por el uso de las tecnologías asociado en fortalecer y aumentar su capital social, logrando ser más eficientes en las actividades que realizan (Kerras et al., 2020), por otro lado también se ha demostrado que el capital social mediante el uso de redes sociales está asociado con el éxito profesional, considerando que una importante actividad con fines profesionales en las redes sociales tiene una fuerte relación con dicho éxito; por lo tanto todo depende del uso y gestión empleados en estos medios, logrando impulsar su trayectoria y experiencia (Nikitkov y Sainty, 2014). También se evidencia que las mujeres profesionales tienen una alta percepción de utilidad de las tecnologías digitales; sin embargo (Cabana et al., 2020) sostiene que en los puestos jerárquicos superiores se observa poca iniciativa e innovación en el uso de estas áreas, es importante mencionar que las competencias digitales también deben estar relacionadas a la competencia emocional y la comunicación efectiva como habilidades que les permitirán potenciarse hacia la empleabilidad, todas ellas orientadas en el uso y manejo de las redes sociales y otras tecnologías interactivas.

Los beneficios de las tecnologías dependen de su uso, ya que se muestran como herramientas que facilitan la comunicación, ayudan a reducir las barreras de espacio y tiempo, mejoran la calidad de vida, convirtiéndose en valiosos recursos para un aprendizaje sostenible, por ello las competencias digitales pueden definirse como la capacidad de utilizar la tecnología eficazmente para optimizar la vida cotidiana considerándose 
además como un compendio de destrezas, habilidades y actitudes ante diferentes áreas y dimensiones de conocimiento (Rodríguez et al., 2019). Las principales áreas de la competencia digital incluyen: 1) conocimiento general y habilidades funcionales de uso de la tecnología, 2) comunicación y colaboración, 3) información, 4) privacidad, seguridad y aspectos legales, 5) uso recurrente y resolución de problemas, convirtiéndose en pilares indispensables para fortalecer el aprendizaje y desempeño de las personas promoviendo su flexibilidad y resiliencia ante diversos cambios o situaciones que pueden hacer llegar al conocimiento a lugares que de otra manera sería imposible, promoviendo acciones vinculadas al desarrollo profesional, uso creativo (Cateriano et al., 2021).

En cuanto a la brecha digital de género, para Botella et al. (2019) se ha venido reduciendo por la marcada participación y uso de las tecnologías móviles, por lo que las desigualdades se enmarcan más bien a aspectos relacionados con la experiencia, habilidades, autonomía y tipos de uso es decir que los usos profesionales junto con la creación de contenidos digitales es lo que permite el logro de habilidades digitales avanzadas. Actualmente se puede apreciar una participación más activa de las mujeres en el diseño de las tecnologías de información en todas las etapas, logrando subsanar un déficit significativo en la construcción del capital humano y una sociedad más tecnológica e inclusiva. Es importante tomar en cuenta que tanto hombres como mujeres son los principales actores en el desarrollo de la sociedad del conocimiento, por tanto, es importante considerar la libre participación de los géneros en buscar beneficios más sostenibles y perdurables a través del tiempo, siempre fomentando un acceso y respeto de los derechos humanos y la lucha de oportunidades (Fountain, 2000).

La revolución digital nace del conjunto de las innovaciones, nuevos cambios tecnológicos en la producción enmarcados por el acceso y uso masivo al Internet, robótica y plataformas digitales entre otros (Nikitkov y Sainty, 2014). Estos cambios han logrado una transformación en los mercados, percepción de nuevas necesidades, incrementos y creación de nuevos empleos, eliminación de trabajos tradicionales, por lo que la formación profesional en nuevos ámbitos es trascendental para poder vincularse en los nuevos desafíos que requieren las sociedades modernas (Kerras et al., 2020). Sin embargo, la diferencia entre hombres y mujeres referente al acceso de las TIC se puede ver en muchas sociedades, tanto en los países económicamente avanzados como en los países que se encuentran en vías de desarrollo.

Según Mumporeze y Prieler (2017) los motivos por los cuales el género femenino no dedica mucho tiempo para acceder a las TIC, son: escasez de tiempo, falta de recursos económicos, priorización en educación, vestido, alimentación de sus hogares para miembros cercanos de su familia, lo que en cierta forma limita a las mujeres contar con oportunidades de aprendizaje, en donde la inversión se focaliza en otras áreas, afectándolas en la inserción y desempeño en el ámbito laboral, otro de los aspectos que impide esta participación e inclusión en los espacios de ciencia, tecnología e innovación , dentro de un contexto de cambio productivo orientado hacia la consolidación de la sociedad del conocimiento está asociado con la promulgación y ejecución de acciones afirmativas y normativas políticas impulsadas a través de los gobiernos de turno, quienes deben ser capaces de integrar una perspectiva de género en los proceso de TIC, consolidando acciones que permitan, garanticen y promuevan la participación de las mujeres en áreas más trascendentes donde puedan potenciar sus conocimientos, capacidades y destrezas para su beneficio personal y en favor de las organizaciones donde generan estos cambios, mecanismos y leyes indispensables que tanta falta hacen en el desarrollo e impulso de las sociedades actuales (Monroy-Merchán, 2019).

Las competencias y habilidades en el género son trascendentales en los diversos ámbitos personales y empresariales, permitiendo que destrezas, conocimientos y actitudes puedan vincularse en diferentes entornos, en función de la demanda de la sociedad y de una forma óptima, por ello (Rojas-Flores, 2018) sostienen tres niveles de competencia: 1) Competencia de base: relacionada al acceso a lugares de trabajo en función del idioma, perfil profesional o el uso de las TIC; 2) Competencia técnica: refiere a conocimientos especializados para un determinado puesto de trabajo; 3) Competencia transversal: que ayuda a la ejecución del trabajo, se enfoca en el ámbito laboral.

Las competencias digitales de género se originan en entornos digitales de acuerdo a los avances tecnológicos y el nivel educativo, lo que a su vez promueve un incremento y fortalecimiento de las competencias profesionales y digitales, permitiendo el acceso a puestos jerárquicos más altos en el caso de los hombres, mientras que en las mujeres sus competencias son menores debido al limitado desarrollo digital, lo que resulta contraproducente para acceder a cualquier cargo y optar por ascensos laborales (Levano-Francia, 2019).

Para (Martínez, et al., 2018) las competencias digitales deben existir en dos niveles: de conocimiento y uso; en el primer nivel de información el usuario realiza búsquedas y consultas referente a temas específicos, y el segundo nivel transaccional, el usuario puede realizar transacciones en línea, generar formularios, solicitudes, entre otros, lo que ayudará en el incremento de su productividad, eficiencia y oportunidades, por lo expuesto se puede evidenciar la importancia de los niveles sin distinción de género, lo que a su vez promoverá una igualdad y equiparación de funciones y responsabilidades, las cuales siempre deben estar en una 
capacitación continua para lograr mayor competitividad en las diferentes áreas laborales (Jaramillo, 2015). Por lo expuesto el objetivo de la presente investigación es conocer el nivel de competencias digitales que poseen los directivos de Gobiernos Autónomos Descentralizados en torno al conocimiento y manejo de herramientas digitales básicas para su gestión en función del género y sus actitudes hacia las TIC.

\section{METODOLOGÍA}

La población o universo para el desarrollo de la investigación estuvo conformado por 85 personas (68,24\% hombres y $27,06 \%$ mujeres); se tomó en consideración directores y jefes departamentales de tres Gobiernos Autónomos Descentralizados de la ciudad de Ibarra. No se realizó ningún cálculo muestral y se trabajó con toda la población existente.

Se empleó una metodología cuantitativa, con un método no experimental, descriptivo e inferencial, y la compilación de información se realizó mediante un cuestionario en soporte electrónico que fue presentado a criterio de tres expertos del área de Tecnología quienes validaron el instrumento conformado por 86 preguntas. Los bloques 1 y 2 fueron de selección múltiple. El bloque 3 sobre conocimiento y manejo de dispositivos, se utilizó la escala de Likert con ítems valorados de 1 a 10; de igual forma, el bloque 4 acerca de la actitud se consideró una valoración desde 1 -muy en desacuerdo- hasta 5 -muy de acuerdo-.

El análisis de las competencias estudiadas se realizó a partir de los diferentes ítems estructurados en cuatro bloques generales: Bloque 1. Identificación (ID). Datos de identificación: género, edad, puesto de trabajo, título profesional. Bloque 2. Posesión (PS). Dispositivos TIC que poseen: equipos, conectividad e instrumentos para el desempeño de sus actividades. Bloque 3. Conocimiento y manejo (CM). Autoevaluación de conocimientos sobre conceptos, uso de herramientas y servicios relacionados con las TIC en seis ejes: 1) Gestión de la información; 2) Comunicación y Colaboración Digital; 3) Trabajo y liderazgo en Red; 4) Aprendizaje continuo; 5) Visión Estratégica; 6) Seguridad. Bloque 4. Actitud (AC). Valoración de las TIC de acuerdo con su necesidad e importancia para desempeño profesional.

Para comprobar la consistencia interna de la encuesta, se utilizó Alfa de Cronbach, cuyo coeficiente alcanzó el valor de 0,8951; valoración que corrobora la fiabilidad del instrumento. La tabulación de los datos y el análisis descriptivo de los mismos se realizó por medio del paquete estadístico SPSS v22.0. Los datos fueron analizados con estadística descriptiva básica e inferencial a partir de una comparación de medias utilizando las pruebas no paramétricas de U Mann - Whitney para la variable dicotómica género.

\section{RESULTADOS Y DISCUSIÓN}

El grupo de empleados analizados corresponde a servidores públicos, quienes lideran departamentos y trabajan con un equipo de personas en la toma de decisiones. De estos el $90 \%$ poseen título de cuarto nivel, acorde a su función, con edades que varían a partir de 32 a 58 años; sin embargo, la brecha de género en cuanto a ocupación de puestos de trabajo de directivos está marcada por el $68,24 \%$ de hombres y $27,06 \%$ mujeres.

En cuanto a la posesión de dispositivos TIC (equipos, conectividad, uso de internet y demás instrumentos) que poseen los servidores públicos en su lugar de trabajo para el desempeño de sus actividades diarias manifiestan el $53 \%$ de los empleados contar con un adecuado equipo tecnológico que incluyen programas actualizados acorde a su desempeño laboral, mientras el $47 \%$, consideran que los equipos son rancios para su necesidad. El dispositivo mayormente utilizado según Figura 1, en un $33 \%$ es el ordenador y la portátil con el $26 \%$. En cuanto a la conectividad la Figura 2 muestra que el $81 \%$ posee conexión a Internet desde su trabajo; sin embargo, la Figura 3 evidencia que el $46 \%$ utiliza el servicio de 6 a 8 horas diarias en su lugar de trabajo; el $56 \%$ lo hace en menos horas ya que tienen que salir de oficina. Las herramientas más utilizadas por los directivos son las ofimáticas (28\%) y las especializadas (38\%), por el tipo de actividad que realizan de acuerdo con el cargo como se observa en la Figura 4. Por ende, se coincide con (Mayor-Buzón et al., 2019) que es fundamental contar con equipos, instrumentos y garantías de calidad, con índices óptimos de validez y fiabilidad para generar una gestión eficiente y responsable en los directivos de la administración pública.

Del conjunto de competencias digitales analizadas, los servidores públicos se destacan en 16 de ellas, en las cuales se obtuvo puntuaciones por encima del valor medio de la escala como se muestra en la Figura 5. Sobre conocer el nivel de competencia digital de los servidores públicos de rango directivo, los datos obtenidos indican una media de 5,63 y una desviación típica de 1,485 que corresponde a un nivel medio de competencia digital. 


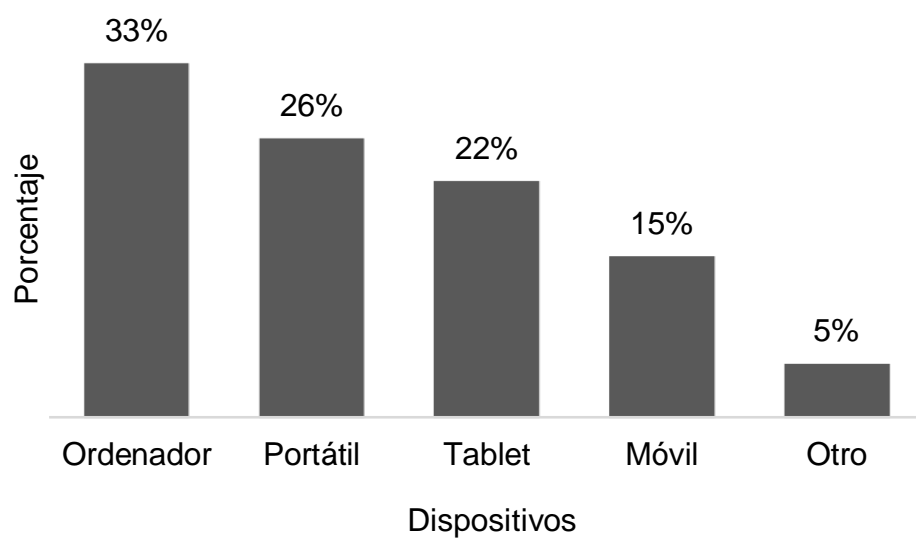

Fig.1: Porcentaje de uso de dispositivos

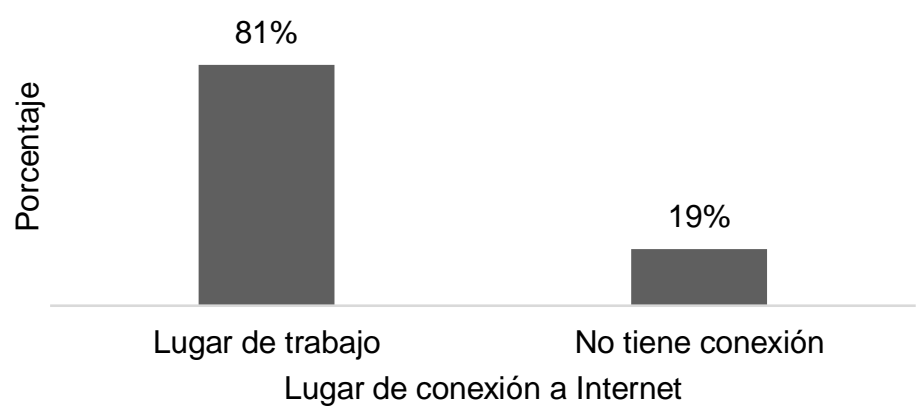

Fig. 2: Porcentaje de conexión a Internet en su trabajo

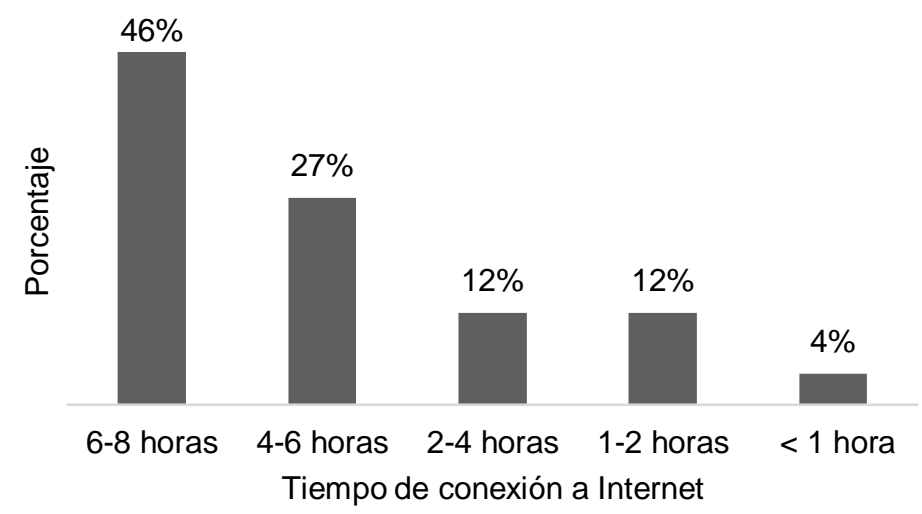

Fig. 3: Porcentaje de horas de conexión a Internet

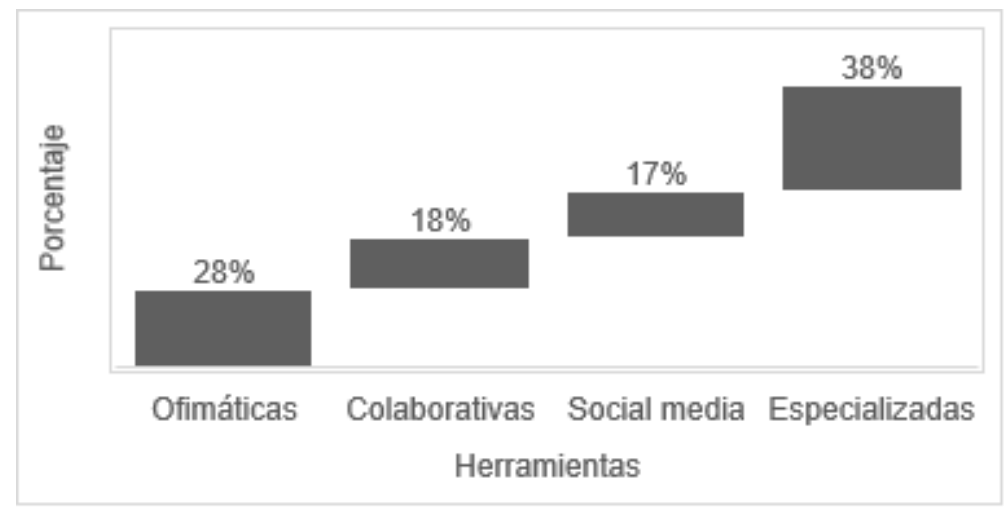

Fig. 4: Porcentaje de herramientas digitales utilizadas 
En cuanto a gestión de la información que utilizan estrategias de navegación, búsqueda y obtención de información $(8,69)$ y tienen la capacidad de compartir información en contextos digitales dentro y fuera de su oficina $(7,84)$; referente a comunicación y colaboración digital poseen la capacidad para colaborar en la web en plataformas como: MSOficce365, G-Suite $(7,49)$ y de comunicarse con diversas herramientas en línea como: WhatsApp, Telegram, e-mail, entre otras, asociadas a relacionar y colaborar de forma eficiente con una adecuada gestión de identidad digital con un puntaje promedio de $(6,37)$, el uso de dispositivos móviles para manejar las redes sociales (8,51). Así se afirma lo que (Carlisle et al., 2021) referente a las habilidades digitales futuras más importantes serán habilidades de comunicación, habilidades en redes sociales, habilidades de MS Office, habilidades de uso de sistemas operativos y habilidades para monitorear reseñas en línea.

En relación con el trabajo y liderazgo en red poseen escasas habilidades al generar redes con agentes internos y externos a la organización $(3,5)$. El mismo resultado se puede notar en la capacidad de trabajar de forma colaborativa, compartiendo recursos para generar mayor valor con otras organizaciones mediante el uso de herramientas web $2.0(4,5)$; sin embargo, se evidencia que manejan agendas digitales, realizan gestión de calendarios para sus reuniones $(7,94)$; comparten conocimiento $(6,77)$, realizan buenas prácticas de bienestar digital $(6,96)$. Por otra parte, se evidenció poco interés en el aprendizaje continuo, $(4,75)$ aunque las mujeres acentuaron mayor actitud a gestionar su propio aprendizaje de manera autónoma, conocer y utilizar recursos digitales y estar constantemente actualizadas. Con respecto a su visión estratégica dentro de la orientación de proyectos no consideran a las TIC como un eje estratégico para una mejor gestión.

Por otro lado, las seguridades, dependen significativamente del departamento de Tecnologías de la Información (TI) aunque por iniciativa propia se sienten capaces de instalar un antivirus, tomar medidas preventivas y correctivas de seguridad en sus dispositivos electrónicos: como uso y cambio de contraseñas y de protegerse en las redes $(5,82)$, en este último aseguran contar con el apoyo humano del departamento de TI. La figura 5, describe las competencias sobre el promedio en las cuales se destacaron en su evaluación los servidores públicos.

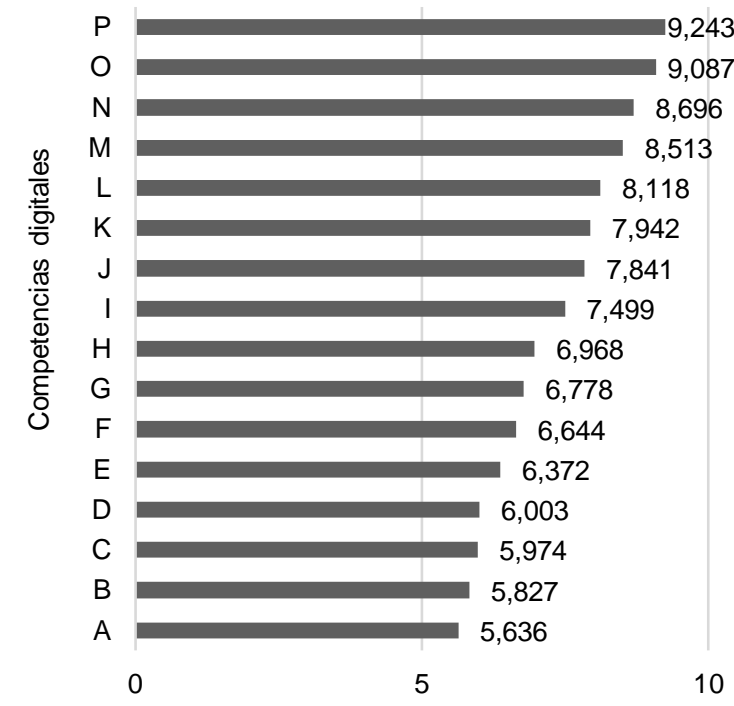

Puntaje alcanzado sobre el promedio
A) Utilizo normas de netiqueta en entornos digitales.

B) Uso y actualizo antivirus y sistemas de seguridad digital.

C) Utilizo herramientas para recuperar archivos.

D) Estoy familiarizado con las herramientas digitales.

E) Uso herramientas de comunicación en línea.

F) Uso y proceso información de manera crítica y sistemática.

G) Comparto conocimiento con otros profesionales conectando a través de la red.

H) Conozco y aplico normas de bienestar digital.

I) Uso herramientas de colaboración en línea.

J) Gestión y almacenamiento online.

K) Uso y comparto un sistema de gestión de calendarios digital.

L) Resuelvo incidencias técnicas sencillas como la instalación de programa.

M) Uso de redes sociales.

N) Aplico estrategias de navegación por internet.

O) Realizo configuración sencilla de dispositivos digitales.

P) Toma medidas de ahorro de energía con los dispositivos electrónicos.

Fig. 5: Ranking de competencias digitales de los directivos de Gobiernos Autónomos Descentralizados.

Referente a su experiencia en el uso de las TIC para el desarrollo de sus actividades en el lugar de trabajo, el $62,23 \%$ afirmó usarlas de forma frecuente, el $26,77 \%$ de forma moderada y apenas el $5,36 \%$ no las usan por incomodidad o desconocimiento de integración en su lugar de trabajo y otro 5,64\% no respondió. Estos resultados coinciden parcialmente con estudios previos como el de Piñeiro (2020) quien ha demostrado que una mayor intensidad y diversidad de uso de las tecnologías incide en un mayor nivel de competencia digital. Pues cerrar esta brecha de habilidades tecnológicas permite que el personal que ya está en la fuerza de trabajo se vuelva digitalmente empoderado, apoye la innovación digital interna y proporcione conjuntos de habilidades digitales sostenibles para todos los niveles de personal (Carlisle et al., 2021).

En concordancia con Rebollo-Catalán et al., (2017), quienes manifiestan existir diferencias entre hombres y mujeres en el uso de herramientas digitales y advierten de la presencia de otra brecha digital de género que 
implica la limitada habilidad y autonomía de uso de las tecnologías en las mujeres cuyas diferencias son el resultado de la desigualdad en las actitudes, percepciones y confianza de hombres y mujeres respecto al uso de las TIC. Esta investigación evidencia que la variable diferenciadora de género demuestra que los hombres tienen mayor conocimiento y manejo de las herramientas digitales con respecto a las mujeres así expresa la Figura 6, pues manejan de mejor manera los dispositivos, herramientas y servicios, incluso fuera de su oficina ya que poseen mejor comunicación por medios digitales que las mujeres. Sin embargo, otros estudios revelan que las mujeres se han incorporado de forma más activa al uso de las redes sociales que los hombres, lo que supone el aumento de su presencia y participación digital, así como su empoderamiento en red.

Para el análisis de las diferencias de conocimiento y manejo de las TIC entre hombres y mujeres (CN) mediante la Tabla 1. se asevera lo planteado en la hipótesis 1 de que los hombres presentan una mayor competencia digital que las mujeres, en este caso se pudo verificar la existencia de diferencias en función del género, es decir, se confirma que los resultados rondan la significación estadística mediante la aplicación de la prueba $U$ de Mann-Whitney, como significativos referentes al bloque de $C N(p=0,165)$ de donde se demuestra que los hombres poseen mayor conocimiento y manejo de las TIC que las mujeres.

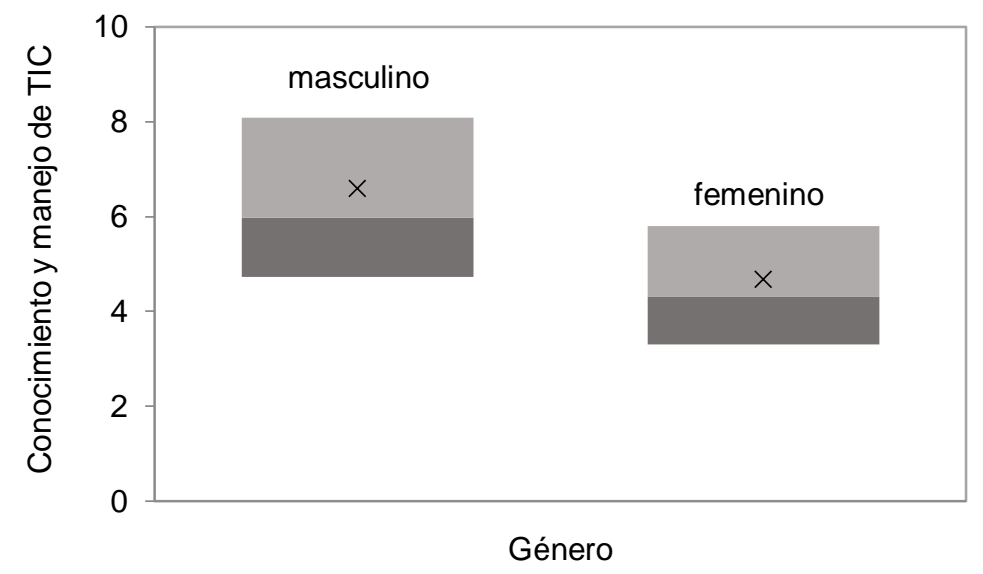

Fig. 6: Diferencias de conocimiento y manejo de las TIC entre hombres y mujeres

Tabla 1: Análisis inferencial de género sobre el conocimiento en TIC

\begin{tabular}{|c|c|c|c|c|c|c|c|c|}
\hline$C N=$ conocimiento & $v c c$ & $u$ & $z$ & $s d$ & $\bar{X}$ & $p$ & Modelo & Resultados \\
\hline Masculino & Sí & \multirow{2}{*}{109800} & \multirow{2}{*}{-2119} & 2,25 & 7,22 & \multirow{2}{*}{0,165} & No Paramétrico: & $P>0,05$ \\
\hline Femenino & Sí & & & 1,81 & 5,31 & & U Mann-Witney & Se acepta la hipótesis planteada \\
\hline
\end{tabular}

Concerniente a la dimensión de actitudes hacia las TIC, los servidores públicos consideran estar muy de acuerdo que las TIC son mayoritariamente interesantes y potencian el aprendizaje $(4,7)$, son accesibles e imprescindibles (4,01). Sobresale el alto porcentaje de interés que tienen las mujeres por actualizar sus conocimientos en TIC $(4,4)$. La Figura 7 expresa que los hombres son quienes poseen mejor condición hacia las TIC que las mujeres, pero con una mínima diferencia que implica una buena actitud para fortalecer sus conocimientos y habilidades en el manejo de herramientas digitales.

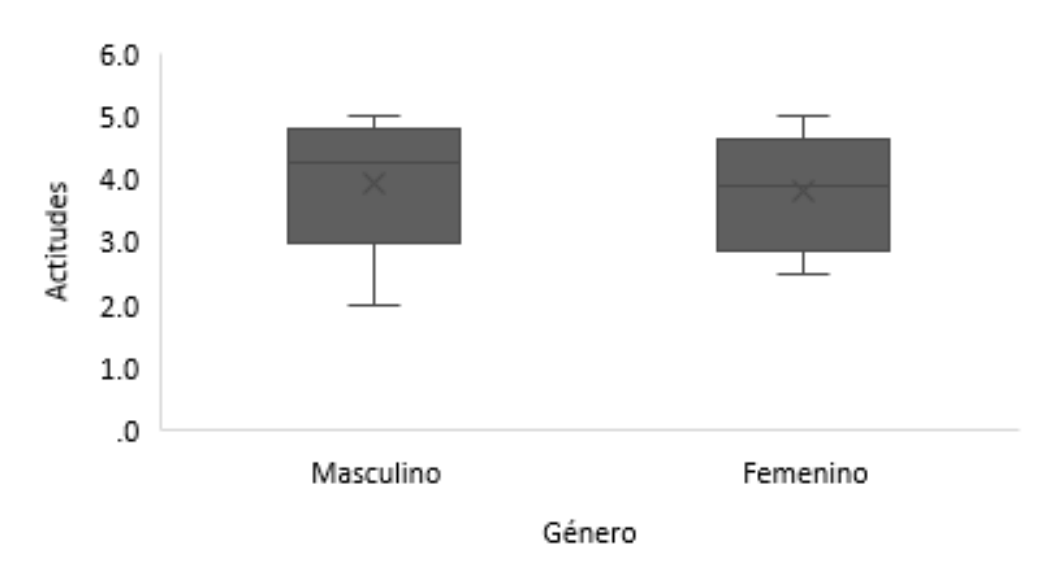

Fig. 7: Actitudes hacia las TIC de hombres y mujeres 
La tabla 2 muestra que existe una relación positiva sobre la actitud ante las TIC entre hombres y mujeres que confirma la Hipótesis 2 en cuanto a que los hombres presentan una mayor actitud hacia las TIC que las mujeres. Esto concuerda con Mayor-Buzón et al., (2019) quienes han identificado la actitud hacia las tecnologías como uno de los factores con mayor incidencia en las habilidades y destrezas que las personas desarrollan en torno con el Internet, es decir se demuestra una relación entre la actitud y competencia.

Tabla 2: Análisis inferencial de género sobre la actitud hacia las TIC.

\begin{tabular}{|c|c|c|c|c|c|c|c|c|}
\hline$A C=$ actitud & $v c c$ & $u$ & $z$ & $s d$ & $\bar{x}$ & p. & Modelo & Resultados \\
\hline Masculino & Sí & & & 1,36 & 4,82 & & & $P>0,05$ \\
\hline Femenino & Sí & 269180 & 1014 & 1,10 & 3,61 & 0,281 & Mann-Whitney & Se acepta la hipótesis planteada \\
\hline
\end{tabular}

El personal directivo del sector público cuenta con tareas que conllevan a decisiones de gran responsabilidad encaminadas al logro de objetivos (Al-Maani et al.,2020), sin embargo, su principal dificultad no es solo burocrática sino de su propia actitud y de apoyo a sus gobernantes. Afortunadamente esta investigación pudo valorar una adecuada condición e interés de los servidores públicos de uso y medición de conocimiento en cuanto a tecnología. En ese sentido Cabana et al., (2020) considera que para cumplir eficientemente se requiere también de aptitudes que destaquen habilidades, capacidades y conocimientos que lleven a cabo con éxito, determinada actividad; esto conjuga con una eficiente gestión pública responsable que depende también de la calidad y eficiencia de las políticas donde no dejen de lado el uso de herramientas tecnológicas para fortalecer un gobierno digital a la altura de una ciudadanía que busca eficiencia y buen vivir con los servicios del sector público.

\section{CONCLUSIONES}

De los resultados mostrados, del análisis y discusión, se pueden obtener las siguientes conclusiones: 1) En los puestos directivos sigue prevaleciendo la presencia de hombres con respecto a las mujeres, resaltándose que ambos llegan a una formación profesional adecuada, pues cuentan con títulos de cuarto nivel. 2) En cuanto al uso de dispositivos para la información y comunicación, las diferencias de género no son significativas, pero muestran conocimientos de uso y servicios con respecto a la idoneidad para el desempeño, es claro que deben mejorar ya que laboran en instituciones públicas. 3) La visión estratégica, liderazgo, trabajo en red y aprendizaje continuo son aspectos más predominantes en el género masculino; 4) La actitud y valoración de las tecnologías de la información referente a necesidades e importancia revela una incorporación más activa de los hombres respecto a las mujeres. 5) Se evidencia un progreso considerable por parte del género femenino en cuanto a las brechas entre hombres y mujeres.

\section{AGRADECIMIENTOS}

Este trabajo forma parte del proyecto de investigación "Competencias digitales empresariales y brecha tecnológica” financiado por la Universidad Técnica del Norte-Ecuador.

\section{REFERENCIAS}

Abreu-Valdivia, O., Rhea-González, S., Arciniegas-Romero, G., y Guevara-López, S., Competencia para el diseño y la ejecución del proceso de enseñanza-aprendizaje en la Facultad de Ciencias Administrativas y Económicas, Universidad Técnica del Norte de Ecuador, https://doi.org/10.4067/S0718-50062020000400153, Formación Universitaria 13(4), 153164 (2020)

Al-Omari, Z.S., Alomari, K.A., y Aljawarneh, N.M., The Role of empowerment in improving internal process, customer satisfaction, learning and growth, https://doi.org/10.5267/j.msl.2019.10.013, Management Science Letters, 10(4), 841848 (2020)

Al-Maani, A.I., Al-Adwan, A., y otros tres autores., Level of administrative empowerment at private institution and its impact on institutional performance: a case study, https://doi.org/10.9770/jesi.2020.8.2(30), Entrepreneurship and Sustainability Issues, 8(2), 500-511 (2020)

Botella, C., Rueda, S., López, E., y Marzal, P., Gender diversity in stem disciplines: a multiple factor problem, https://doi.org/10.3390/e21010030, Entropy, 21(1), 30 (2019)

Cabana, S.R., Cortés, F.H., Contreras, F.A., y Vargas, V.F., Influencia del control de gestión al valor público generado en servicios dependientes del ministerio de economía fomento y turismo Chile, https://doi.org/10.4067/S071807642020000200103, Información Tecnológica, 31(2), 103-116 (2020) 
Cabezas González, M., Casillas Martín, S., Sanches-Ferreira, M., y Teixeira Diogo, F. L., ¿Condicionan el género y la edad el nivel de competencia digital? un estudio con estudiantes universitarios,

https://doi.org/10.14201/fjc201715109125, Fonseca: Journal of Communication, 15(15), 109-125 (2017)

Carlisle, S., Ivanov, S., y Dijkmans, C., The digital skills divide: evidence from the European tourism industry, Journal of Tourism Futures, https://doi.org/10.1108/JTF-07-2020-0114, 27 (2021)

Cateriano-Chávez, T.J., Rodríguez-Ríos, M.L., y otros tres autores, Competencias digitales, metodología y evaluación en formadores de docentes, Campus virtuales, 10(1), 153-162 (2021)

Fountain, J.E., Constructing the Information society: women, information technology and design, https://doi.org/10.1016/S0160-791X(99)00036-6, Technology in Society, 22(1), 45-62 (2000)

Gilch, P.M., y Sieweke, J., Recruiting digital talent: the strategic role of recruitment in organizations digital transformation, https://doi.org/10.1177/2397002220952734, German Journal of Human Resource Management, 35(1), 53-82 (2021)

Jaramillo, O., Pertinencia del perfil de los profesionales de la información con las demandas del mercado laboral, Revista Interamericana de Bibliotecología, 38, 111-120 (2015)

Kerras, H., Sánchez, J., Erasmo, I., y López-Becerra, M., The impact of the gender digital divide on sustainable development: comparative analysis between the European Union and Maghreb, https://doi.org/10.3390/su12083347, Sustainability, 12(8), 33-47 (2020)

Khalayleh, W.A., Masa'deh, D.R., y Al-Lozi, P.M., Administrative empowerment and its role on the work teams performance: a literature review, https://doi.org/10.25255/jss.2017.6.4.851.868, Journal of Social Sciences, 6(4), 851-868 (2017)

Levano-Francia, L., Sánchez-Diaz, S., y otros cuatro autores, Competencias digitales y educación, https://doi.org/10.20511/pyr2019.v7n2.329, Propósitos y Representaciones, 7(2), 569-588 (2019)

Martínez-Bravo, M., Charo-Sábada, C., y Serrano-Puche, J., Desarrollo de competencias digitales en comunidades virtuales: un análisis de "ScolarTIC", Prisma Social, 20, 129-159 (2018)

Mayor-Buzón, V., García-Pérez, R., y Rebollo-Catalán, A., Explorando factores predictores de la competencia digital en las redes sociales virtuales, Píxel-Bit Revista de Medios y Educación, https://doi.org/10.12795/pixelbit.2019.i56.03, 56, 51-69 (2019)

Monroy-Merchán, M.L., Sociedad del conocimiento y las brechas de género en ciencia, tecnología e innovación, https://doi.org/10.18270/cuaderlam.v15i29.2871, Cuadernos Latinoamericanos de Administración, 15(29), 1-12 (2019)

Mumporeze, N., y Prieler, M., Gender digital divide in Rwanda: a qualitative analysis of socioeconomic factors, https://doi.org/10.1016/j.tele.2017.05.014, Telematics and Informatics, 34(7), 1285-1293 (2017)

Nikitkov, A., y Sainty, B., The role of social media in influencing career success, International Journal of Accounting and Information Management, 4, 273-294 (2014)

Pérez-Escoda, A., Lena-Acebo, F. J., y García-Ruiz, R., Brecha digital de género y competencia digital entre estudiantes universitarios, https://doi.org/10.17811/rifie.50.1.2021.505-5014, Aula Abierta, 50(1), 505-5014 (2021)

Piñeiro, C., Recursos TIC y supervivencia empresarial: una revisión de la noción de ventaja competitiva, Estudios de Economía, 47(1), 79-125 (2020)

Rebollo-Catalán, Á., Mayor-Buzón, V., y García-Pérez, R., Competencias digitales de las mujeres en el uso de las redes sociales virtuales: diferencias según perfil laboral, https://doi.org/10.6018/rie.35.2.270881, Revista de Investigación Educativa, 35(2), 427-444 (2017)

Rodríguez, A., Raso, F., y Ruiz, J., Competencia digital, educación superior y formación del profesorado: un estudio de meta-análisis en la Web of Science, https://doi.org/10.12795/pixelbit.2019.i54.04, Pixel-Bit Revista de Medios y Educación, 54(1), 65-82 (2019)

Rojas-Flores, A.R., Rojas-Salazar, A.O., y otros tres autores, Aplicación del módulo alfabetización digital y desarrollo de competencias digitales en docentes, Comuni@cción, 9(2), 101-109 (2018)

Sebastian, C.M., y Ayuso, M., Situación de la brecha digital de género y medidas de inclusión en España, https://doi.org/10.22201/iibi.0187358xp.2011.55.32973, Investigación Bibliotecológica: Archivonomía, Bibliotecología e Información, 25(55), 227 (2012)

Trenerry, B., Chng, S., y otros cinco autores, Preparing workplaces for digital transformation: an integrative review and framework of multi-level factors, https://doi.org/10.3389/fpsyg.2021.620766, Frontiers in Psychology, 12(1), 1-24 (2021)

Vasilescu, M.D., Serban, A.C., y otros tres autores, Digital divide, skills and perceptions on digitalisation in the European Union-Towards a smart labour market, https://doi.org/10.1371/journal.pone.0232032, PLoS ONE 15(4), 1-18 (2020) 
